\title{
Wireless SET: An Approach for the Deployment of SET over WAP Mobile Networks
}

\author{
Alia Fourati, Hella Kaffel Ben Ayed, Farouk Kamoun \\ Abdelmalek Benzekri \\ Laboratoire CRISTAL, ENSI, Université de la Manouba, Tunis, Tunisia \\ Laboratoire IRIT, Université Paul Sabatier, Toulouse, France
}

\begin{abstract}
This paper addresses the deployment of the Secure Electronic Transaction (SET) protocol for the payment in the mobile commerce. The strength of this protocol is in its capability to separate within the same message, the order information intended to the seller and the payment information intended to the payment gateway. We note that the payment information must be seen only by the payment gateway. Moreover, this approach avoids the "WAP gap" since the payment information would not be decrypted within the WAP gateway.
\end{abstract}

\section{INTRODUCTION}

This work explores the possibility of deploying the SET protocol in the mobile commerce. We propose an approach, called WSET (Wireless SET), securing payment in the m-commerce. We prove that WSET allows the avoiding of some SET limits by exploiting the possibilities of WTLS / TLS protocols of the WAP. In addition, it will make it possible to avoid the problem of the "WAP gap" on the WAP1.X. This paper falls into three parts:

- Section I presents the SET protocol.

- Section II presents the payment in the m-commerce.

- And finally, section III describes the WSET approach.

\section{THE SET PROTOCOL PRESENTATION}

The SET (Secure Electronic Transaction) protocol has been defined to guarantee the security of credit card payment through Internet [1] and [4].

The original version of this chapter was revised: The copyright line was incorrect. This has been corrected. The Erratum to this chapter is available at DOI: 10.1007/978-0-387-35620-4_43 


\subsection{SET Operation Principle}

The SET standard defines a set of messages which are exchanged between the software of the purchaser, the merchant and the payment gateway.

The SET protocol defines the 4 following phases:

\section{The Registration Phase Of The Customer And The Merchant}

The SET protocol imposes to each communicating party to have a SET certificate. The registration phase requires the exchange of six messages for each party, allowing thus the authentication of the credit card holder, the merchant and the payment gateway by SET digital certificates.

The Purchase Request Phase

Once a given buyer is interested by a product, he sends a purchase request to the seller implying the exchange of 4 messages [4]. These messages include the certificate exchange between the buyer and the seller. They also contain a transaction identifier generated by the seller to protect against the transaction replay. The buyer creates (OI)' and (PI)' from OI (Order Information), PI (Payment Information) and the dual signature of OI and PI. The Dual signature of OI and PI is illustrated in equation 1 below. He finally generates a symmetric key $\mathrm{K} 1$ to encrypt (PI)' and the client credit card number. $\mathrm{K} 1$ is itself encrypted with the public key of the payment gateway $\mathrm{P}_{\mathrm{PpubK}}$. This allows the payment information to go through the seller without being decrypted by him.

The Payment Authorization Request Phase

The payment authorization request phase requires the exchange of 2 messages between the seller and the payment gateway.

The Payment Settlement Request Phase

The payment settlement phase implies the exchange of 2 messages between the seller and the payment gateway.

Dual Signature $=\{\mathrm{MD} 3[\mathrm{MD} 1(\mathrm{OI})+\mathrm{MD} 2(\mathrm{PI})]\}_{\text {BuyerPrivateKey }}$ Where MD1, MD2 and MD3 are three different keyed hash functions.

\subsection{SET Advantages And Drawbacks}

The SET protocol ensures the authentication of the intervening parties and the information confidentiality between the concerned parties in only one signed transaction by the use of the buyer and the payment gateway certificates. The purchase request requires then only one message which contains two fields (OI and PI), the first is intended to the seller and the second is intended to the payment gateway. 
The data integrity is guaranteed by the dual signature. This allows the seller and the payment gateway, independently one of the other to check the integrity of OI for the seller and respectively PI for the payment gateway.

The SET protocol allows protection against the transaction replay by the use of a unique transaction identifier id.

The barrier to the deployment of the SET protocol is especially with the heaviness of the certification chain which slows down the transaction process [2]. In addition, the SET protocol forces the client to have a certificate specific to each credit card he owns.

Finally, SET has the disadvantage of exchanging cryptographic secret keys on line. The messages transmitting these keys are obviously encrypted by the public keys of receivers, but that presents nevertheless attack risks.

\section{THE PAYMENT IN THE M-COMMERCE}

During the payment phase of a mobile commerce transaction, the credit card number must go through the WAP (Wireless Application Protocol) gateway. This gateway has on the one hand to adapt the types of information forwarding in two different environments (wireless and wired), and on the other hand to translate the data format generated by the mobile devices (WML) towards the web servers data format (HTML) and vice versa. This translation relates to all WAP layers. We are interested in our work in the data security layers, namely the TLS layer in the Internet side and the WTLS layer in the wireless network side.

In versions $1 . X$ of WAP, the security of transactions is ensured by the WTLS (Wireless Transport Layer Security) layer. During a WTLS session, there is a negotiation of the security parameters. There is however a security gap on the level of the WAP gateway which was called the "WAP gap" [5], [6] and [7]. In fact, when the encrypted data arrive at the gateway, they are decrypted, then re-encrypted again with TLS (Transport Layer Security) and finally conveyed to the server. During a laps of time, the data are exposed without least security. For the electronic payment, the risk to expose unprotected credit card number of a client can be unacceptable.

\section{THE WIRELESS SET SOLUTION PRINCIPLE}

We propose to use the strong points of SET to ensure a secure payment in the m-commerce environment. We will avoid the weak points of SET by exploiting WTLS/TLS layer services. We will focus on the messages 
exchanged between the buyer and the seller, since they are involved in the wireless and wired environments.

From the SET protocol, we will use:

1. The dual signature principle to ensure the integrity.

2. The transaction identifier generated by the seller.

3. The end to end exchange of the payment gateway certificate.

The operation principle of this approach is illustrated in figure 1.

\subsection{The Scenario Description}

The deployment of the SET protocol on WAP 1.X falls into 3 phases:

- Phase 1: The certificate exchange and the negotiation of the security parameters.

- Phase 2: The purchase request and the using of negotiated cryptographic public keys.

- Phase 3: The payment authorization and settlement between the seller and the payment gateway having the same treatment as in "classic SET ".

\section{Phase 1: Certificate exchange and Negotiation of security parameters} phase

(1) The buyer begins this phase by opening a secure session with the seller. He proposes to the seller server a list of the security parameters he supports. This list includes cryptographic key exchange methods, encryption algorithms, hash algorithms, etc.

(2) The seller answers the buyer by returning an Initialization_Request_S composed of five messages. These messages include the selected security parameters list and a session identifier, the seller and the payment gateway certificates and a buyer certificate request message.

(3) The buyer answers by returning an Initialization_Response_B and_Finished composed of five messages. These messages include the buyer certificate message, a message setting the master secret and an encrypted Finished message verifying the previous security processes.

(4) The seller sends an Initialization_Response_S_and_Finished which is composed of two messages, ending then the phase1.

\section{Phase 2: Purchase request phase}

(5) During this phase, the buyer sends a message similar to the "classic SET" purchase request message. The only difference is that OI will be encrypted with the Ks session key generated from the master secret (shared secret). PI remains encrypted by the payment gateway public key transmitted in the payment gateway certificate.

Phase 3: Payment authorization and settlement phase

Messages (6), (7), (8), (9) and (10) of the payment authorization and settlement phase are identical to those of "classic SET ". 
Wireless SET : An Approach for the Deployement of SET ...

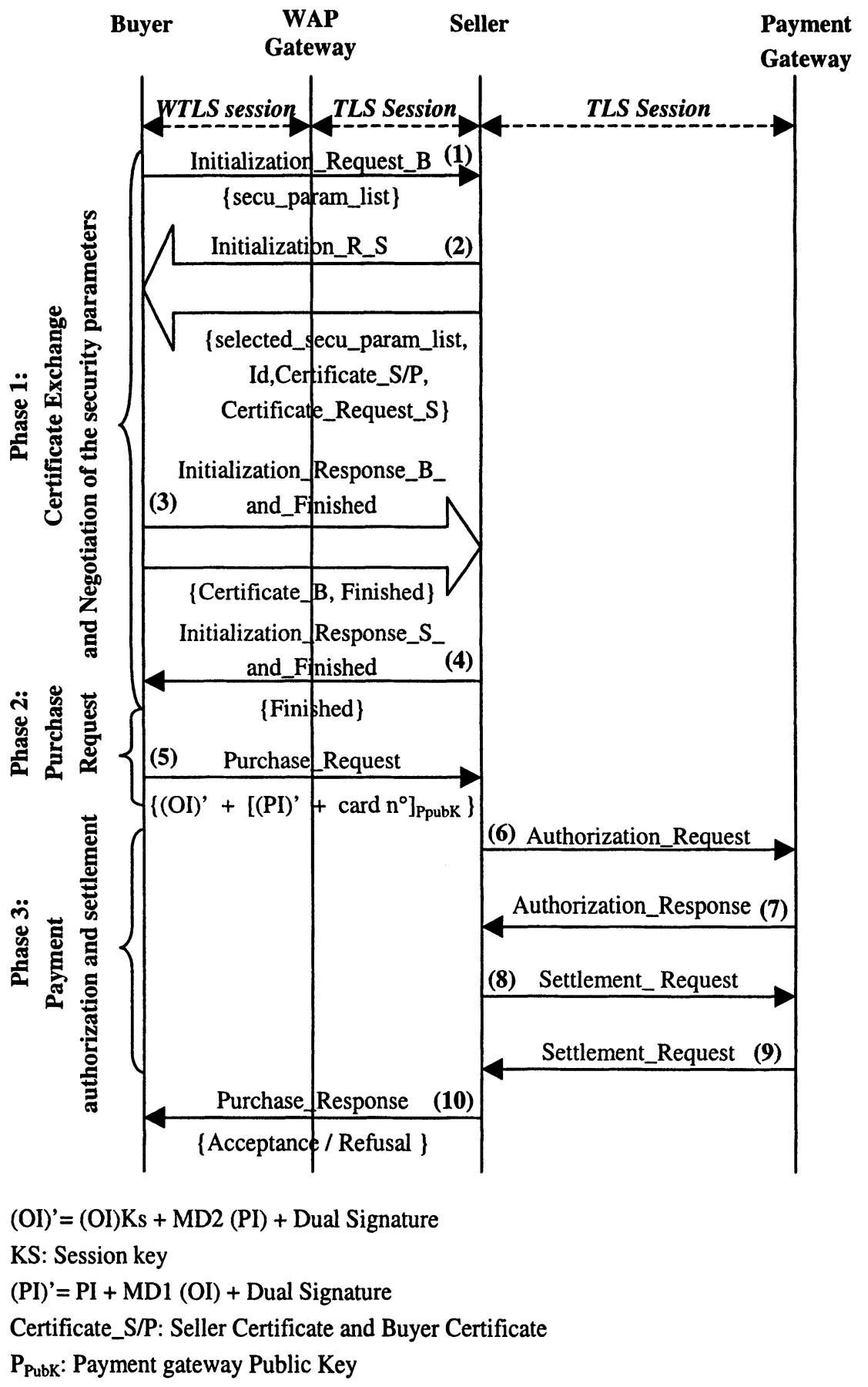

Figure 1. The Scenario of wireless SET on WAP 1.X 


\subsection{The SET Contribution On WAP 1.X:}

The advantages provided by the deploying of SET on the WAP 1.X are: - The separation of the useful data from the encryption data and the providing of an end to end confidentiality of the payment data (which also avoids the "WAP gap").

- The transaction id generated by SET is linked with the SET transaction, which allows end to end integrity and protection against the seller replay attacks. This is not verified with the session_id generated by TLS/WTLS protocols, because it is local to the WTLS session between the mobile client and the WAP gateway and to the TLS session between the WAP gateway and the seller server.

- We avoid the SET certification process too heavy for a mobile environment [2], [4] and [7]. For this reason, we can leave the certification to the task of the WTLS/TLS layers. This enables the intervening parties to use the same certificate for various operations and even with different credit cards.

\section{CONCLUSION}

In this paper, we have explored the deployment of the SET protocol on WAP 1.X. We recommended the use of WTLS certificates to avoid the SET certification heaviness and to enable the clients to use the same certificate for all their commercial transactions. Furthermore, this solution makes it possible to avoid the "WAP gap". In the second time, we have exploited the negotiation of the security parameters provided by the WTLS/TLS protocols. This allows to separate the user data from the encryption data and to introduce more flexibility to the SET protocol.

\section{REFERENCES}

1. Garfinkel, Simson and Spafford, Gene, "Web Security, Privacy \& Commerce ". O'Reilly, 2002.

2. Ben Ayed, Hassan, "Approche de sécurité pour le commerce électronique basée sur le protocole IPsec ”. ENSI, CRISTAL Lab, 2001.

3. WAPForum, "Wireless Transport Layer Security Specifications”. Version April 06, 2001. http://www.wapforum.org

4. SET consortium, "SET Specifications". May 31, 1997. http://www.setco.org

5. Durham-Vichr, Deborah and Getgen, Kimberley, "Securing the Internet without wires". IBM developer works, aout 2001.

http://www-106.ibm.com/developerworks/library/wi-sectrends/index.html

6. Saarinen, Markku-Juhani, "Attacks against the WAP WTLS Protocol". University of Jyvaskyla. Finland. http://www.jyu.fi/ mjos/wtls.pdf

7. Howell, Ric, "WAP Security”. Concise Group Ltd. http://www.vbxml.com/wap/articles/wap-security/default.asp 


\section{TRAFFIC ENGINEERING AND TCP}

